\title{
Current status of treatment for colorectal liver metastases in the United Kingdom
}

\author{
Kentaro Okamoto, Hiroyuki Uetake \\ Graduate School, Tokyo Medical and Dental University, Tokyo, Japan \\ Correspondence to: Hiroyuki Uetake, MD, PhD. Professor, Department of Specialized Surgeries; Director, Department of Chemotherapy and \\ Oncosurgery, Graduate School, Tokyo Medical and Dental University; 1-5-45, Yushima, Bunkyo-ku, Tokyo, 113-8519, Japan. \\ Email: h-uetake.srg2@tmd.ac.jp. \\ Comment on: Fenton HM, Taylor JC, Lodge JPA, et al. Variation in the Use of Resection for Colorectal Cancer Liver Metastases. Ann Surg \\ 2019;270:892-8.
}

Submitted Feb 28, 2020. Accepted for publication Apr 09, 2020.

doi: $10.21037 / \mathrm{hbsn} .2020 .04 .08$

View this article at: http://dx.doi.org/10.21037/hbsn.2020.04.08

Hepatectomy is the treatment choice for resectable colorectal cancer (CRC) liver metastases (LM) (CRCLM) according to the national guidelines (1-3). The National Comprehensive Cancer Network guidelines indicate that the treatment for liver-limited metastases depends on the resectability (1). In the European Society for Medical Oncology guideline, a similar statement is made on the treatment of resectable CRCLM, except for the recommendation of perioperative chemotherapy for some populations with resectable CRCLM (2). In the Japanese Society for Cancer of the Colon and Rectum (JSCCR) guidelines, hepatic resection is recommended for LMs if all the lesions are curatively resectable (3). The indication criteria for hepatectomy in the JSCCR guidelines are as follows: (I) the patient can tolerate surgery; (II) the primary tumor has been controlled or can be controlled; (III) the metastatic liver tumor can be completely resected; (IV) extrahepatic metastases are absent or can be controlled; and (V) the function of the remaining liver will be adequate (3). The authors of the present study commented in the introduction that outcomes of CRC in the UK are known to be behind those in many economically comparable countries, maybe because of the poorer outcomes in patients with advanced disease at the first visit to hospital. They also indicated that previous studies showed that the resection rates of CRCLM showed significant variation across the English National Health Service (NHS), which indicates the possibility of a considerable variation in the decision-making process to decide the treatment strategy for CRCLM with or without a specialist liver team. Thus, the aim of the current study was to investigate the rate of hepatic resection for CRCLM across the English NHS.

As a result, authors identified 157,383 patients who underwent a major resection for a colorectal tumor between January 1, 2005, and December 31, 2012. Of the 7,423 $(4.7 \%)$ patients who underwent 1 or more hepatectomy within 3 years after the primary CRC was resected, $89.9 \%$ had 1 resection, $9.3 \%$ had 2 resections, and $0.8 \%$ had 3 or more resections. Most $(90.4 \%)$ of the patients received their first hepatectomy after the primary CRC was removed, of whom $6.7 \%$ had a synchronous hepatectomy with the primary CRC resection and $2.9 \%$ had their first hepatectomy performed before the resection of primary CRC. The liver-first strategy (4), which precedes LM prior to primary resection, is not performed often in the UK.

The percentage of patients who underwent hepatectomy increased between 2005 and 2012. During the last 4 years of the study period, it reached a stable figure up to approximately $5 \%$ for all patients with primary CRC resection. On the other hand, the Charlson comorbidity index of the all the patients in the present study increased over time. The proportion of patients with 1 or more comorbidities increased from $22.8 \%$ to $25.2 \%$ during the course of the study period. Although patients aged $>70$ years made up $51.4 \%$ of those who underwent a CRC resection, they accounted for only $29.4 \%$ of those who underwent hepatectomy. In the $>70$ s group, frequency of hepatectomy increased more than doubled over time, from $1.2 \%$ in 
$1998-2004$ to $2.8 \%$ in $2005-2012$. The fact suggests that management patterns may have changed. These data suggest that hepatectomy is expected to be safely performed for patients with complications or elderly patients in recent years.

Patients who underwent primary CRC resection in a hospital with a specialist liver team were more likely to underwent hepatectomy (odds ratio, 1.22; 95\% confidence interval, 1.10-1.35). The authors speculated that it is due to a higher frequency of hepatectomy performed synchronously to resection of primary CRC resection in these patients (18\%) than in those who received primary CRC resection in a hospital without a specialist liver team in the same hospital (3\%). Patients who received the primary CRC resection in a hospital with a specialist liver team onsite were $22 \%$ more likely to underwent hepatectomy than those who had to be consulted to other hospitals. This result suggests that referral pathways may not be as efficient as they should be. Patients may lose the access to treatment, depending on their proximity to a hospital with a specialist liver team. In other word it is suggested that proximity to a liver center is as an important socioeconomic deprivation factor in terms of the low likelihood of receiving a liver resection.

The frequency of major hepatectomy (hemihepatectomies and extended hemihepatectomies) was reduced over the study period from $48.2 \%$ to $39.9 \%$ $(\mathrm{P}<0.001)$. The major hepatectomy rate was $36.1 \%$ in a hospital with a specialist liver team and $41.1 \%$ for patients who underwent primary CRC resection in hospitals without a specialist liver team $(\mathrm{P}<0.001)$. For multiple lesions, hepatectomy may be performed at once. However, the theoretical advantage of a policy for preserving the liver parenchyma and major vessels as much as possible was reported in preparation for repeated resections in case of another recurrence (parenchyma-preserving hepatectomy) $(5,6)$. Oba et al. reported that repeated hepatectomy is actively performed for recurrence in approximately $70 \%$ of cases after resection of CRCLM and that the therapeutic effect of hepatectomy did not diminish regardless of whether it was performed repeatedly (7). The development of major hepatectomy procedures such as liver partition and portal vein ligation for staged hepatectomy (ALPPS) (8) has been reported, although it is limited to the general clinical practice in the UK.

In CRC chemotherapy, targeted therapy with a high tumor shrinkage effect has been performed since the early 2000s. Recently, the treatment of metastatic CRC (mCRC) has remarkably progressed with biological agents. Nowadays, the median survival time of patients with mCRC is more than 30 months. Under such circumstances, identifying the biological agent preferred for treatment, especially as first-line treatment of mCRC in patients with RAS wild-type tumor, becomes more and more important (9). For unresectable mCRC, the preferred treatment should be decided by considering the treatment goal; patients should be treated to attain maximum shrinkage, or to be maintained treatment duration without severe adverse event. For the former, anti-EGFR monoclonal antibody (mab) might be a preferred option in terms of depth of response. For the latter, bevacizumab (BEV) might be preferred for treatment maintenance without severe side effect. Oki et al. reviewed clinical trials of the effectiveness of chemotherapy for CRCLM (10). For unresectable liver-limited diseases, a similar treatment strategy can be recommended. LM is of several types. If the LM is bulky and unresectable, tumor shrinkage is needed to convert the LM to resectable. If the LM is disseminated, a pathological effect can prevent recurrence after liver resection. For the former, anti-EGFR mab maybe a better treatment option. For the latter, BEV may be better, considering the characteristics of the mabs $(9,11)$. A preferred treatment option should be considered according to a real clinical case (11).

In the TRICC0808 trial, we showed that mFOLFOX6 plus BEV therapy was followed by a high hepatectomy rate and a high conversion rate (12). In the trial, the rate of chemotherapy-associated adverse events and hepatectomy complications were both acceptable. In the ATOM trial, a randomized phase II study of mFOLFOX6 with BEV compared with mFOLFOX6 with cetuximab (CET) for CRCLM that is upfront unsuitable for resection, we reported that BEV and CET showed similar efficacies in terms of progression-free survival and liver resection rate, although greater tumor shrinkage was observed in the CET group (13).

In this study, the rate of hepatectomy in women was low even when the tumor location was corrected. In CRC, genetic characteristics such as the consensus molecular subtypes are becoming clearer (14). The treatment result of CRCLM may be affected by sex-based genetic background.

\section{Acknowledgments}

Funding: None. 


\section{Footnote}

Provenance and Peer Review: This article was commissioned by the editorial office, Hepatobiliary Surgery and Nutrition. The article did not undergo external peer review.

Conflicts of Interest: Both authors have completed the ICMJE uniform disclosure form (available at https://hbsn. amegroups.com/article/view/10.21037/hbsn.2020.04.08/coif). The authors have no conflicts of interest to declare.

Ethical Statement: The authors are accountable for all aspects of the work in ensuring that questions related to the accuracy or integrity of any part of the work are appropriately investigated and resolved.

Open Access Statement: This is an Open Access article distributed in accordance with the Creative Commons Attribution-NonCommercial-NoDerivs 4.0 International License (CC BY-NC-ND 4.0), which permits the noncommercial replication and distribution of the article with the strict proviso that no changes or edits are made and the original work is properly cited (including links to both the formal publication through the relevant DOI and the license). See: https://creativecommons.org/licenses/by-nc-nd/4.0/.

\section{References}

1. NCCN National Comprehensive Cancer Network (NCCN) guideline for colon cancer. Available online: https://www.nccn.org/professionals/physician_gls/pdf/ colon.pdf

2. Van Cutsem E, Cervantes A, Adam R, et al. ESMO consensus guidelines for the management of patients with metastatic colorectal cancer. Ann Oncol 2016;27:1386-422.

3. Hashiguchi Y, Muro K, Saito Y, et al. Japanese Society for Cancer of the Colon and Rectum (JSCCR) guidelines 2019 for the treatment of colorectal cancer. Int J Clin Oncol 2020;25:1-42.

4. Andres A, Toso C, Adam R, et al. A survival analysis of the liver-first reversed management of advanced simultaneous colorectal liver metastases: a LiverMetSurvey-based study.
Ann Surg 2012;256:772-8; discussion 778-9.

5. Pawlik TM, Choti MA. Surgical therapy for colorectal metastases to the liver. J Gastrointest Surg 2007;11:1057-77.

6. von Heesen M, Schuld J, Sperling J, et al. Parenchymapreserving hepatic resection for colorectal liver metastases. Langenbecks Arch Surg 2012;397:383-95.

7. Oba M, Hasegawa K, Shindoh J, et al. Survival benefit of repeat resection of successive recurrences after the initial hepatic resection for colorectal liver metastases. Surgery 2016;159:632-40.

8. Schnitzbauer AA, Lang SA, Goessmann H, et al. Right portal vein ligation combined with in situ splitting induces rapid left lateral liver lobe hypertrophy enabling 2 -staged extended right hepatic resection in small-for-size settings. Ann Surg 2012;255:405-14.

9. Uetake H. The Role of Systemic Chemotherapy. In: Kim NK, Sugihara K, Liang JT. editors. Colorectal Cancer. Surgical Treatment of Colorectal Cancer. Asian Perspective on Optimization and Standardization. Singapore: Springer, 2018; 329-44.

10. Oki E, Ando K, Nakanishi R, et al. Recent advances in treatment for colorectal liver metastasis. Ann Gastroenterol Surg 2018;2:167-75.

11. Uetake H, Tanaka S, Ishikawa T, et al. Fate of metastatic foci after chemotherapy and usefulness of contrastenhanced intraoperative ultrasonography to detect minute hepatic lesions. J Hepatobiliary Pancreat Sci 2012;19:509-14.

12. Uetake $H$, Yasuno $M$, Ishiguro $M$, et al. A multicenter phase II trial of mFOLFOX6 plus bevacizumab to treat liver-only metastases of colorectal cancer that are unsuitable for upfront resection (TRICC0808). Ann Surg Oncol 2015;22:908-15.

13. Oki E, Emi Y, Yamanaka T, et al. Randomised phase II trial of mFOLFOX6 plus bevacizumab versus mFOLFOX6 plus cetuximab as first-line treatment for colorectal liver metastasis (ATOM trial). Br J Cancer 2019;121:222-9.

14. Loree JM, Pereira AAL, Lam M, et al. Classifying Colorectal Cancer by Tumor Location Rather than Sidedness Highlights a Continuum in Mutation Profiles and Consensus Molecular Subtypes. Clin Cancer Res 2018;24:1062-72.
Cite this article as: Okamoto K, Uetake H. Current status of treatment for colorectal liver metastases in the United Kingdom. HepatoBiliary Surg Nutr 2021;10(1):116-118. doi: 10.21037/hbsn.2020.04.08 